\title{
EVALUATION AND SUGGESTION OF ENVIRONMENTAL DEGRADATION AND ITS EFFECTS IN AZAMGARH DISTRICT
}

\section{आजमगढ़ जनपद में पर्यावरण अवनयन और उसके प्रभावों का मूल्यांकन एवं सुझाव}

\author{
Dr. Sanjay Kumar Sharma ${ }^{1 凶}$, Raj Narayan Prajapati ${ }^{2}$ \\ ${ }^{1}$ Director of Research, President and Associate, Professor Hindu College, Moradabad, Department \\ of Geography, India \\ 2 Researcher, Geography Department, Hindu College, Moradabad, MA, NET, M.Phil., India
}

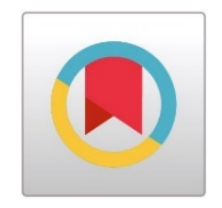

DOI: https://doi.org/10.29121/granthaalayah.v8.i10.2020.1894

Article Type: Research Article

Article Citation: Dr. Sanjay Kumar Sharma, and Raj Narayan Prajapati. (2020). EVALUATION AND

SUGGESTION OF ENVIRONMENTAL DEGRADATION AND ITS EFFECTS IN AZAMGARH DISTRICT.

International Journal of Research GRANTHAALAYAH, 8(10), 172-175. https://doi.org/10.29121/granthaa layah.v8.i10.2020.1894

Received Date: 04 October 2020

Accepted Date: 30 October 2020

Keywords:

आजमगढ़

पर्यावरण

मूल्यांकन एवं सुझाव

\section{ABSTRACT}

English: In the context of the presented research study area Azamgarh district, the evaluation of environmental degradation and its effects, due to the changing activity of variable development between environment and human beings, the current activities only point towards its insensitivity. Increasing problems around our environment such as soil pollution, water pollution, air pollution and biodiversity havoc, future and future crisis on environment and human existence have arisen resulting in climate change increasing natural disasters, drinking water problem, global warming, Increase in incurable diseases of human beings, there are different types of effects of all living organisms including humans, due to which environmental degradation and population growth and industrialization, rapid urbanization, consumerist culture have considered the basic root of environmental degradation. The following suggestions will be presented by the researcher environmental impact and evaluation.

Hindi: प्रस्तुत शोध अध्ययन क्षेत्र आजमगढ़ जनपद के सन्दर्भ में पर्यावरण अवनयन और उसके प्रभावों का मूल्यांकन पर्यावरण एवं मानव के बीच परिवर्तनशील विकास के बदलते क्रियाकलाप के कारण वर्तमान गतिविधियाँ उसकी असंवेदनशीलता की ओर ही इशारा करती है। हमारे वातावरण के आसपास बढ़ती समस्याएं जैसे मृदा प्रदूषण, जल प्रदूषण, वायु प्रदूषण एवं जैव विविधता का तीव्र ह्नास , पर्यावरण एवं मानव अस्तित्व पर भविष्य के लिए संकट उत्पत्र हो गया जिसके परिणाम स्वरूप जलवायु परिवर्तन प्राकृतिक आपदाओं में वृद्धि, पेयजल की समस्या, वैश्विक उष्मन, मानव के असाध्य रोगों में वृद्धि, मानव सहित सभी जीवधारियों के विभित्र प्रकार के प्रभाव हैं जिसका कारण-पर्यावरण अवनयन एवं जनसंख्या वृद्धि तथा औद्योगीकरण, तीव्र नगरीकरण, उपभोक्तावादी संस्कृति ने पर्यावरण अवनयन का मूल जड़ माना जा रहा है। शोधार्थी पर्यावरण प्रभाव एवं मूल्यांकन के द्वारा निम्नलिखित सुझाव को प्रस्तुत किया जायेगा।

\section{1. प्रस्तावना}

अध्ययन क्षेत्र आजमगढ़ जनपद उत्तर प्रदेश राज्य के पूर्वांचल क्षेत्र में भौगोलिक स्थिति $25028^{\prime}$ से $26027^{\prime}$ उत्तरी अक्षांश तथा $82041^{\prime}$ से $83052^{\prime}$ पूर्वी देशान्तर के मध्य स्थित है जो घाघरा, सरयू तथा गोमती नदी के मध्य गंगा के मैदान में एक घना हुआ जनपद है। जनपद का कुल

(C) 2020 The Author(s). This is an open access article distributed under the terms of the Creative Commons Attribution License, which permits unrestricted use, distribution, and reproduction in any medium, provided the original author and source are credited. 


\section{आजमगढ़ जनपद में पर्यावरण अवनयन और उसके प्रभावों का मूल्यांकन एवं सुझाव}

भौगोलिक क्षेत्रफल 4054 वर्ग किमी० है । पूर्व से पश्चिम की लम्बाई 75 किमी०, उत्तर से दक्षिण की लम्बाई 85 किमी है। 2011 की जनगणना के अनुसार कुल जनसंख्या 4612134 है, जिसमें 22 लाख पुरूष तथा 23 लाख से अधिक महिलाए है। जनसंख्या घनत्व 1138 प्रति वर्ग किमी० हैं। लिंगानुपात 1000/1019 महिलाऐं तथा जनवृद्धि 15.82 प्रतिशत हैं। साक्षरता 72.92 प्रतिशत है। "अध्ययन क्षेत्र जनपद आजमगढ़ वैदिक काल से ऋषियों एवं मुनियों, सन्तों आदि की पुण्य भूमि रही है। यह घने जंगलों में बसा हुआ था तथा दुर्बासा ऋषि तमसा (टौंस) नदी के पुनीत तट पर तपस्या किया था, जनपद का उत्तरी भाग सरयू नदी के तट का दक्षिणी भाग है जो हर वर्ष अगस्त से सितम्बर माह बाढ़ से प्रभावित रहता है तथा दक्षिणी भाग गोमती नदी का उत्तरी भाग कंकरीला, ऊसरीला, अनुपजाऊ क्षेत्र के रूप में बसा हुआ है। अध्ययन क्षेत्र के मध्य भाग में तमसा नदी के द्वारा निर्मित मैदान बना हुआ है जो कृषि फसलों के उत्पादन के लिए प्रसिद्ध है।"

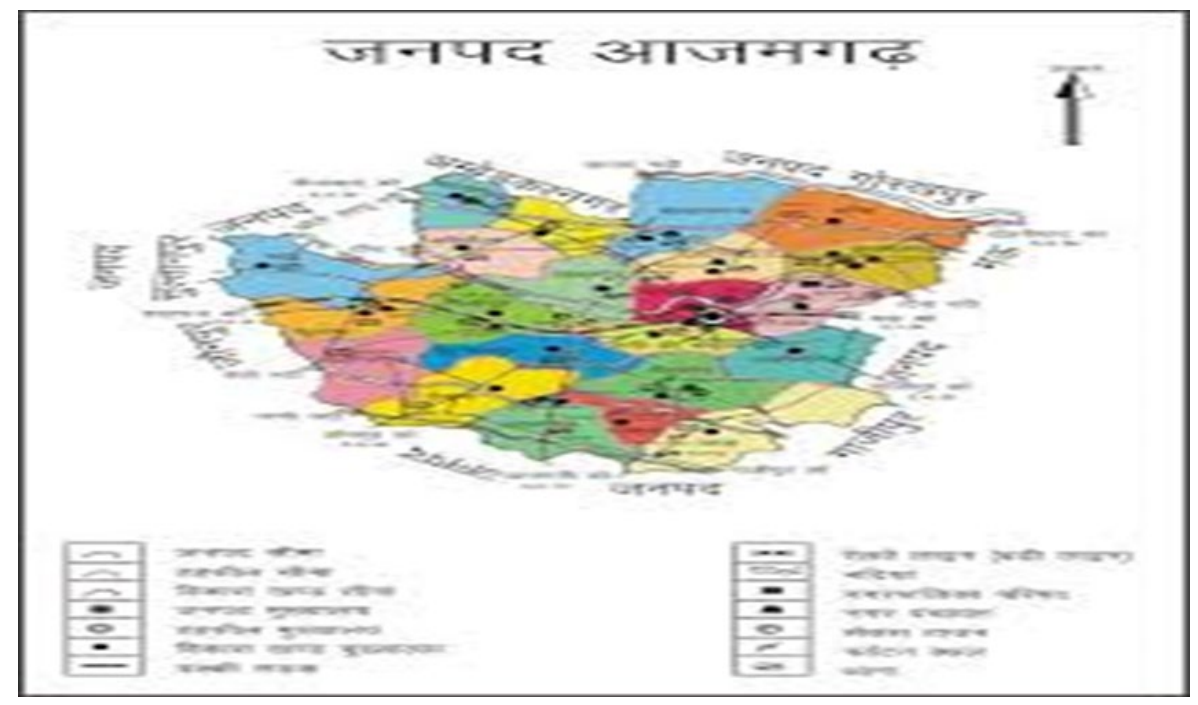

\section{2. पर्यावरण अवनयन साहित्यावलोकन}

पर्यावरण अवनयन से तात्पर्य पर्यावरण के विभित्र घटकों यथा मृदा, वायु, जल तथा जैवविविधता के वास्तविक गुणों एवं विशेषताओं में होने वाले अवांछनीय परिवर्तन से है। प्रकृति में सामान्य परिस्थितियों में अवनयन एवं निर्माणकारी प्रक्रियाएँ एक चक्रीय रूप से घटित होती रहती है व उनमें सन्तुलन की स्थिति बनी रहती है। " पर्यावरण अवनयन मनुष्य के क्रियाकलापों द्वारा पर्यावरण के संघटकों की आधारभूत संरचना में प्रतिकूल परिवर्तनों के कारण पर्यावरण की गुणवत्ता में इस सीमा तक ह्नास होना कि इन प्रतिकूल परिवर्तनों का जैविक समुदाय तथा मानव समाज पर गहरा प्रतिकूल प्रभाव पड़ने लगता है। पर्यावरण अवनयन के कारण पारिस्थितिक तंत्र एवं पारिस्थितिकी की विविधता में कमी होने से पारिस्थितिकीय एवं पर्यावरणीय असन्तुलन उत्पन्न हो जाता है।

पर्यावरण पृथ्वी पर वह प्रवृत्ति है जो मानव को चारों ओर से घेरे हुए है अर्थात् हमारे चारों ओर प्रकृति में जो दिखाई देता है जैसे जल, वायु, मिट्टी, पौधे, प्राणी तथा समस्त दशाए मनुष्य को किसी न किसी रूप में प्रभावित करती है। मानव जीवन जिन दशाओं पर निर्भर रहता है। भौतिक, जैविक, सांस्कृतिक प्रक्रियाओं के कारण पर्यावरण के तत्वों में कमी एवं वृद्धि के अवनयन की समस्या उत्पत्र होती है। "भौतिक संसाधनों का प्रयोग मानव के ज्ञान, विज्ञान एवं तकनीकी विकास पर आधारित रहा है जब तक प्रकृति के सहयोग से मानव अपनी आवश्यकता की पूर्ति करता रहा, तब तक पर्यावरण सन्तुलन बना रहा है लेकिन आधुनिक समाज में बढ़ती हुई जनसंख्या बढ़ता भौतिकवाद, उच्च तकनीकी और प्रकृति के प्रति व्यवहार की प्रगति की रफ्तार ने संसाधन ,दोहन, अत्यधिक ऊर्जा प्रयोग, औद्योगिकीकरण, शहरीकरण ,सोर मण्डलीय क्रियाकलाप ने पर्यावरण असन्तुलन की स्थिति उत्पत्र किया है।"

आज सम्पूर्ण विश्व में विकास की अन्धी प्रवृत्ति के पर्यावरण को असन्तुलित करती जा रही है। इसी का परिणाम चक्रवात, बाढ़, सूखा, भूस्खलन, वनों में आग लगने की घटनाए दिन-प्रतिदिन बढ़ती जा रही है। "प्रकृति आपदाओं से नुकसान को कम करने के लिए संयुक्त राष्ट्र ने पिछले दशक ( 1990-1999) को अन्तर्राष्ट्रीय प्राकृतिक आपदा न्यूनीकरण दशक के रूप में बनाया है। भारत सरकार प्राकृतिक आपदा दिवस अक्टूबर का दिन राष्ट्रीय आपदा न्यूनीकरण दिवस के रूप मे मनाने का फैसला किया तथा 1999 में इसी दिन ओडिसा में महाविनाशकारी चक्रवात आया था, जिससे अपार जन-धन की हानि हुई थी। राष्ट्रीय आपदा न्यूनीकरण दिवस के बारे में जागरूकता पैदा करना तथा उनसे निपटने के लिए तैयार रहकर नुकसान कम किया जा सकता है।"

वर्तमान समय में इस तकनीक प्रधान वैश्वीकरण युग में मानवीय गतिविधियों के कारण प्राकृतिक संसाधनों के अतिदोहन से यह चक्र असन्तुलित हो रहा है। भूमि उपयोग प्रतिरूप में परिवर्तन औद्योगीकरण, नगरीकरण की तीव्र दर, उपभोक्तावादी संस्कृति ने पर्यावरण अवनयन की दर को तीव्र गति प्रदान किया है। जिसका परिणाम स्वरूप पर्यावरण असन्तुलन के रूप में सामने दिखाई दे रहे है। "विशेषकर 20 वीं शताब्दी के मध्य के बाद में पर्यावरणीय समस्याओं ने वैश्विक स्वरूप धारण कर लिया है। यही कारण है कि 1987 में पर्यावरण और विकास पर ठतनदज संदक कमीशन द्वारा सतत विकास की संकल्पना प्रस्तुत करते हुए विकास की ऐसी प्रक्रिया पर जोर दिया जो वर्तमान की आवश्यकताओं से समझौता किय बिना भविष्य की जरूरतों की पूर्ति भी कर सके।" 


\section{डॉ. संजय कुमार शर्मा, राज नारायण प्रजापति}

\section{3. अध्ययन क्षेत्र में पर्यावरण अवनयन के प्रभावों का कारण}

अध्ययन क्षेत्र सहित पर्यावरण अवनयन तथा उससे जनित विश्वव्यापी पर्यावरणीय संकट सर्वप्रथम कारण मनुष्य तथा प्राकृतिक पर्यावरण के बीच तेजी से बिगड़ता सम्बन्ध, वास्तव में प्राकृतिक संसाधनों का मानव द्वारा तेजी से विदोहन, प्रौद्योगिकी विकास, तीव्र औद्योगिक एवं नगरीकरण के फलस्वरूप पर्यावरण पर प्रतिकूल प्रभाव दिखाई देने लगे। आर० एफ0 दासमैन ने कहा "मानव दौड़ हाथ में ग्रेनेड लिए बन्दर के समान है कोई यह नहीं जानता है कि वह कब ग्रेनेड से पिन खीच लेगा तथा विश्व तहस-नहस हो जायेगा।" इस प्रकार शोधार्थी अपने शोध अध्ययन क्षेत्र सहित सम्पूर्ण विश्व के सन्दर्भ में पर्यावरण अवनयन के प्रभावों के कारणों को निम्न बिन्दुओं द्वारा स्वष्ट किया गया है।

\section{1. जनसंख्या वृद्धि एवं पर्यावरण अवनयन}

शोध अध्ययन सहित विश्व में बढ़ती जनसंख्या व इसके परिणामस्वरूप विभित्र गतिविधियों से पर्यावरण प्रदूषित हो रहा है। शोध क्षेत्र आजमगढ़ जनपद में तीव्र जनसंख्या वृद्धि के कारण मानवीय क्रियायें प्राकृतिक संसाधनों ,तीव्र गति से विदोहन के फलस्वरूप पर्यावरण अवनयन की समस्या बढ़ती जा रही है। जिससे खाद्यात्र में कमी, आवास की समस्या आदि "पर्यावरण पर जनसंख्या वृद्धि के ऐसे कुप्रभाव जैसे पर्यावरण अवनयन को समझने से पहले पारिस्थितिकी मण्डल की संकल्पना भली-भाॅति समझ लेना आवश्यक है। ऐसा प्रतीत होता है कि बढ़ती जनसंख्या वृद्धि पर चिन्ता करनी होगी और भविष्य में विश्व जनसंख्या वृद्धि को नियन्त्रित करके हम पर्यावरण अवनयन की समस्या काफी हद तक कम कर सकते है।

\section{2. वन विनाश एवं पर्यावरण अवनयन}

अध्ययन क्षेत्र में वन सम्पदा किसी राष्ट्र की धरोहर होती है क्योंकि वनों से उद्योगों के लिए कच्चे माल, भवन निर्माण सामग्री, हवा, जीव-जन्तु के आवास, वर्षा, कार्बन डाई आक्साॅड का सुरक्षा कवच आदि । यह घोर चिन्ता का विषय है कि अध्ययन क्षेत्र आजमगढ़ कुल क्षेत्रफल का 248 हे० भूमि वन पाये जाते है। कभी यह धरातल वैदिक काल में वनों घना वसा हुआ है लेकिन मानव के आर्थिक क्रियाकलाप दिन प्रतिदिन वनो का विनाश करते जा रहे है और पर्यावरणीय अवनयन में वृद्धि होती जा रही है। शोधार्थी जनपद एवं विकासखण्ड स्तर पर वनों के घटते क्षेत्रफल तथा पर्यावरण की बढ़ती समस्या से अवगत अध्ययन क्षेत्र में जनमानस को अवगत कराना चाहता है।

\section{3. कृषि विकास एवं पर्यावरण अवनयन}

शोध क्षेत्र जनपद आजमगढ़ कृषि प्रधान क्षेत्र है। यहाँ की 72 प्रतिशत जनसंख्या कृषि क्षेत्रों में निर्भर और आजीविका तथा रोजगार का साधन है। बढ़ती जनसंख्या के कारण भरण-पोषण के लिए कृषि क्षेत्रों में वन विनाश, मृदा प्रदूषण, जल प्रदूषण, वायु प्रदूषण आदि पर्यावरण अवनयन के कारण रूप कृषि विकास को माना जाता है क्योंकि कृषि विकास के लिए भूमि का शोषण, जल का अधिक दोहन, रासायनिक उर्वरकों का अधिक प्रयोग, कीटनाशक दवाओं का उपयोग , सभी कारक कृषि विकास के लिए माने जाते है। लेकिन सभी विकास के तत्व पर्यावरण अवनयन को बढ़ाने के मूल तत्व माने जाते है।

\section{4. औद्योगिक और नगरीकरण पर्यावरण अवनयन}

अध्ययन क्षेत्र के विकास के लिए औद्योगिक कारखाने अति महत्वपूर्ण माने जाते है और उद्योगों का विकास प्राकृतिक संसाधनों पर निर्भर है। प्राकृतिक संसाधनो का अत्यधिक दोहन के फलस्वरूप पर्यावरणीय समस्या का जन्म होता है। औद्योगिक विकास के फलस्वरूप नगरीकरण का विकास होता है तीव्र नगरीकरण के कारण प्राकृतिक संसाधनों का मानव तीव्र गति से अपनी आवश्यकता से अधिक उपयोग करने लगता है। जिसके फलस्वरूप पर्यावरण अवनयन की समस्या बढ़ने लगती है तीव्र औद्योगिक एवं नगरीकरण के कारण वायु प्रदूषण, जल प्रदूषण, मृदा प्रदूषण एवं मानव विभित्र असाध्य रोग-टी०बी०, दमा, कैंसर, टाइफाइड, मलेरिया, डेंगू, बुखार वायरस संक्रमण बीमारियां आदि बढ़ने लगती है।

\section{5. आधुनिक प्रौद्योगिकी एवं पर्यावरण अवनयन}

आधुनिक समय मानव के विकास प्रौद्योगिकी तकनीकी विकास पर आधारित हो गया है क्योंकि अब वर्तमान आधुनिक कौशल प्रौद्योगिकी मिशन के अन्तर्गत विकास के सभी क्षेत्र तकनीक पर आधारित होते जा रहे है। "प्राकृतिक संसाधनों का अधिकतम विदोहन, तीव्र दर से आर्थिक विकास तथा मानव समाज के भौतिक स्तर पर अत्यधिक वृद्धि, पश्चिमी दुनिया के औद्योगिक देशो में आधुनिक प्रौद्योगिक का वैज्ञानिक विकास करके विकसित राष्ट्र बनते जा रहे हैं लेकिन बढ़ते प्रौद्योगिकी विकास के कारण आज मानव सहित सभी क्षेत्रों में विकास की तीव्र गति को आधार प्रदान किया है जैसेशिक्षा, सामाजिक, राजनैतिक, आर्थिक आदि के द्वारा विकास हुआ है तथा 3-4 दिसम्बर 1984 ई0 घटित भोपाल गैस. त्रासदी तथा 1986 में यूक्रेन चरनोबिल नाभिकीय संयन्त्र फटने से बड़ी संख्या में जन-धन की हानि हुई है।" 


\section{आजमगढ़ जनपद में पर्यावरण अवनयन और उसके प्रभावों का मूल्यांकन एवं सुझाव}

इस प्रकार शोधार्थी अपने शोध अध्ययर्न में पर्यावरण अवनयन के सन्दर्भ में पर्यावरणीय समस्या के प्रति चेतना जागृत किया है। पर्यावरण अवनयन की समस्या अब दिन-प्रतिदिन बढ़ती जा रही है क्योंकि मानव की असीमित आवश्यकता को नियंत्रण में न रखने के कारण पर्यावरणीय अवनयन की समस्या बढ़ रही है। महात्मा गाँधी ने कहा है कि "प्रकृति के अन्दर सभी मानव के भरण पोषण के पर्याप्त साधन है कि मानव की लालची प्रवृत्ति का भरण -पोषण नहीं हो सकता है।"

\section{4. अध्ययन क्षेत्र में पर्यावरण अवनयन को कम करने के लिए सुझाव}

शोध अध्ययन आजमगढ़ जनपद में पर्यावरण अवनयन के प्रभावों का मूल्यांकन करते हुए शोधार्थी द्वारा पर्यावरणीय समस्याओं को कम करने के लिए निम्न सुझाव प्रस्तुत किया गये हैं-

1) प्रस्तुत शोध् में सभी पर्यावरणीय समस्याओं की जड़ तीब्र जनसंख्या वृद्धि है, क्योंकि जनसंख्या ही सभी संसाधनों को गति प्रदान करता है लेकिन जब आवश्यकता से बढ़ने पर घातक होगा इसलिए जनसंख्या वृद्धि को नियंत्रण करके पर्यावरणीय अवनयन की समस्या कम किया जा सकता है।

2) पर्यावरण अवनयन की समस्या को कम करने में वनों का विनाश प्रमुख कारक है, क्योंकि वनों से सभी आवश्यकताओं की पूर्ति होती है। शोधार्थी वनों के विकास के लिए सामाजिक वानिकी कार्यक्रम, वृक्षारोपण, जैविक कृषि अन्य भूमि पर वृक्षों का रोपण काय्र महत्वपूर्ण माना है।

3) जनसंख्या के भरण पोषण के लिए कृषि का विकास आधार है । बिना कृषि विकास से लोगों का भोजन, आवस, कपड़ा आदि की आवश्यकताओं की पूर्ति नहीं की जा सकती है। इसलिए कृषि विकास का पर्यावरण अवनयन पर पड़ने वाले प्रभावों का मूल्यांकन करके टिकाऊ, सपोषण कृषि विकास नीति को अपनाना चाहिए।

4) अध्ययन क्षेत्र में औद्योगिक एवं नगरीकरण की गति धीमी है फिर भी औद्योगिक क्षेत्रों का सभी नियोजित व्यवस्था न होने पर अनेक पर्यावरणीय समस्या उत्पन्न होती है। इस प्रकार उद्योगों एवं नगरीकरण के बीच सन्तुलित कार्य को अपनाकर विकास को आधार प्रदान करें। जिससे पर्यावरणीय समस्याए कम हो सके।

5) मानव के बढ़ते प्रौद्योगिकी तकनीक आर्थिक विकास के आधार है, लेकिन उनका दीर्घकालीन प्रभाव मानव सहित सभी जीवधारियों के लिए अभिशाप भी है, क्योंकि मानव बढ़ते तकनीकी ज्ञान जैसे प्लास्टिक के सामान, इलेक्ट्रानिक यन्त्र, रासायनिक पदार्थ, अणु परीक्षण, संचार के साधन, मूलभूत एवं अपशिष्ट पदार्थ, ठोस अपशिष्ट के द्वारा पर्यावरण अवनयन की समस्या दिन-प्रतिदिन बढ़ती जा रही है जिससे प्राकृतिक तत्व अपने मूल तत्व से समाप्त हो जा सकते है जैसे जल प्रदूषण, मृदा प्रदूषण, वायु प्रदूषण, ध्वनि प्रदूषण, अपशिष्ट प्रदूषण आदि को मानवीय क्रियाओं में सन्तुलन स्थापित करके बचाया जा सकता है।

6) राष्ट्रीय एवं अन्तर्राष्ट्रीय सहयोग से संगठनो के द्वारा पर्यावरण अवनयन की समस्या का समाधान किया जा सकता है।

\section{SOURCES OF FUNDING}

None.

\section{CONFLICT OF INTEREST}

None.

\section{ACKNOWLEDGMENT}

None.

\section{REFERENCES}

[1] डॉ. विनोद त्रिपाठीः आजमगढ़ जनपद का पुरातत्व 2004, प्रा0 लि0 हरदोई पृष्ठ सं0-520

[2] डॉ. सविन्द्र सिंह: पर्यावरण भूगोल का स्वरूप 2019, प्रवालिक पब्लिकेशन, इलाहाबाद, पृष्ठ सं0-370

[3] डॉ. लोकेश श्रीवास्तव: पर्यावरण अध्ययन एवं प्रबन्धन: 2012, शारदा पुस्तक भवन, इलाहाबाद, पृष्ठ सं0 155

[4] डॉ. लोकेश श्रीवास्तव: पर्यावरण अध्ययन एवं प्रबन्धन: 2012, शारदा पुस्तक भवन, इलाहाबाद, पृष्ठ सं0 155

[5] डॉ. सविन्द्र सिंह: पर्यावरण भूगोल, 2009, शारदा पुस्तक भवन, इलाहाबाद, पृष्ठ सं० 370

[6] डॉ. सविन्द्र सिंह: पर्यावरण भूगोल का स्वरूप 2019, प्रवालिक पब्लिकेशन, इलाहाबाद, पृष्ठ सं0-372

[7] डॉ. आर0 सी० चाँदना: जनसंख्या भूगोल, 2017, कल्याणी पब्लिकेशन्स, लुधियाना, पृष्ठ सं0 598

[8] डॉ. सविन्द्र सिंह: पर्यावरण भूगोल का स्वरूप 2019, प्रवालिक पब्लिकेशन, इलाहाबाद, पृष्ठ सं0-385,386 